\title{
A New Algorithm Based on Bernstein Polynomials Multiwavelets for the Solution of Differential Equations Governing AC Circuits
}

\author{
Shweta Pandey ${ }^{1}$, Sandeep Dixit ${ }^{2, *}$ and Sag R Verma ${ }^{1}$ \\ ${ }^{I}$ Department of Mathematics and Statistics, Gurukula Kangri Vishwavidyalaya, Haridwar 249404, India \\ ${ }^{2}$ Department of Mathematics, University of Petroleum and Energy Studies, Dehradun 248007, India
}

('Corresponding author's e-mail: sdixit@ddn.upes.ac.in)

Received: 6 June 2020, Revised: 6 May 2021, Accepted: 6 June 2021

\begin{abstract}
We extend the application of multiwavelet-based Bernstein polynomials for the numerical solution of differential equations governing $\mathrm{AC}$ circuits (LCR and LC). The operational matrix of integration is obtained from the orthonormal Bernstein polynomial wavelet bases, which diminishes differential equations into the system of linear algebraic equations for easy computation. It appeared that fewer wavelet bases gave better results. The convergence and exactness were examined by comparing the calculated numerical solution and the known analytical solution. The error function was calculated and illustrated graphically for the reliability and accuracy of the proposed method. The proposed method examined several physical issues that lead to differential equations.
\end{abstract}

Keywords: AC circuits, Bernstein polynomials multiwavelets, Operational matrix, Wavelet analysis

\section{Introduction}

A differential equation is a mathematical equation that depicts the connection between a function (physical values in the case of a physical model) and its derivatives. Differential equations can be used to solve issues in mathematical physics such as mechanics, elasticity, the linearized theory of water waves, stable steady-state heat conduction, and radioactive heat transfer. AC circuits include features that are used in a variety of everyday gadgets. Many numerical methods based on different operational matrices have been developed to determine solutions of differential equations with a given degree of accuracy [14] Wavelets were also used for solving several physical problems [5-8]. In this paper, we show the application of the proposed method in LCR (electric circuit comprising of resistance (R), capacitor (C) and inductor (L)) and L-C circuits (comprising of inductor (L) and capacitor (C)) circuits see Figure 1. We solved examples based on these circuits.
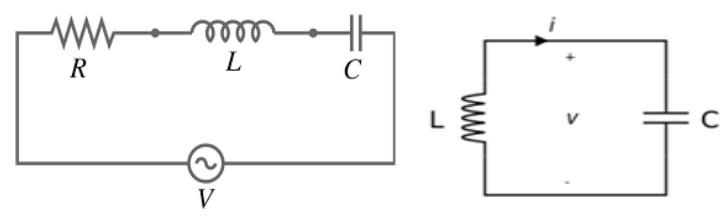

Figure 1 LCR and LC circuit diagram.

The resonance effect of the LC circuit has significant applications in communications systems and signal processing, such as the tuning of radio to a specific radio channel. LC circuits are set at resonance for that specific carrier frequency and used to pick out a signal from that particular radio station at a particular frequency from a more complex signal. Generating signals at that particular frequency is the key component in many applications filters, mixers, oscillators and tuners. The series and parallel resonant circuits give voltage and current magnification, respectively. When subject to a sinusoidal applied voltage $V(t)=V_{0} \sin \omega t$, the differential equation governing the current flow in a series LCR circuit is; 


$$
L \frac{d^{2} q}{d t^{2}}+R \frac{d q}{d t}+\frac{q}{C}=V_{0} \sin \omega t
$$

The differential equation governing the current flow in a series LC circuit when subject to a sinusoidal applied voltage $V(t)=V_{0} \sin \omega t$ is

$$
L \frac{d^{2} i}{d t^{2}}+\frac{1}{C} i=\omega V_{0} \cos \omega t
$$

In this proposed algorithm, a wavelet-based numerical method on Bernstein polynomials multiwavelets to solve the differential equation is given. In this paper, firstly we integrate the given differential equation then remove the integral operators by approximating the given function with the help of the newly constructed operational matrix of integration based on Bernstein polynomials multiwavelets, which converts the above differential equation into a system of algebraic equations for easier computations. The first example describes how the proposed methodology fits for finding a solution of the LCR circuit and the second example describes the LC circuit and 1 general example is given to understand the proposed method better.

\section{Wavelets and Bernstein polynomials multiwavelets}

Wavelets have achieved the present growth as a result of various numerical investigations [9-13]. Wavelets are used to compress given information in a manner so that the resulting signal is a better representation of the information than the given signal was in the original form. The continuous variation of the translation and dilation parameters $u$ and $\mathrm{v}$ gives the following continuous wavelet form [1]

$$
\psi_{u, \mathrm{v}}(t)=|u|^{-1 / 2} \psi\left(\frac{t-\mathrm{v}}{u}\right), \quad u, \mathrm{v} \in R, \quad u \neq 0
$$

when these 2 parameters $u$ and $\mathrm{v}$ are regulated to $u=2^{-d}, \mathrm{v}=n 2^{-d}$ then new discrete wavelet family is obtained from the above equation as $\psi_{d, i}(t)=2^{d / 2} \psi\left(2^{d} t-i\right) ; d, i \in Z$, and $\int_{R} \psi(t) d t=0$. Bernstein polynomials of order $n$ characterized over the interval $[0,1]$ are given as:

$$
B_{m, n}(t)=\left(\begin{array}{c}
n \\
m
\end{array}\right) t^{m}(1-t)^{n-m}, \forall m=0,1,2, . ., n .
$$

Using the Gram-Schmidt process $n+1$ orthonormal Bernstein polynomials for order $n$ can be obtained easily from Bernstein polynomials $B_{m, n}(t)$ given in Eq. (4).

Eight orthonormal Bernstein polynomials of order 7, which are obtained from Bernstein polynomials $B_{m, n}(p)$ given in Eq. (4) by utilizing the Gram-Schmidt process are given below:

$$
\begin{aligned}
b_{0}(t) & =\sqrt{15}(1-t)^{7} \\
b_{1}(t) & =\sqrt{13}(t-1)^{6}(-1+15 t) \\
b_{2}(t) & =-\sqrt{11}(t-1)^{5}\left(1-28 t+105 t^{2}\right) \\
b_{3}(t) & =3(t-1)^{4}\left(-1+39 t-273 t^{2}+455 t^{3}\right) \\
b_{4}(p) & =-\sqrt{7}(t-1)^{3}\left(1-48 t+468 t^{2}-1456 t^{3}+1365 t^{4}\right) \\
b_{5}(p) & =\sqrt{5}(t-1)^{2}\left(-1+55 t-660 t^{2}+2860 t^{3}-5005 t^{4}+3003 t^{5}\right) \\
b_{6}(p) & =\sqrt{3}\left(1-61 t+885 t^{2}-5225 t^{3}+15125 t^{4}-22737 t^{5}+17017 t^{6}-5005 t^{7}\right) \\
b_{7}(p) & =-1+63 t-945 t^{2}-5775 t^{3}-17325 t^{4}+27027 t^{5}-21021 t^{6}+6435 t^{7} .
\end{aligned}
$$


Bernstein polynomials orthonormal multiwavelets $\psi_{i, j}(t)=\psi(d, i, j, t)$ where $d$ is dilation parameter assumes any positive integer, $j=0,1,2 \ldots, \mathrm{N}$ is Bernstein polynomial degree, translation parameter $i=0,1,2, \ldots, 2^{d}-1$, and normalized time $t$. They are characterized on the interval $[0,1)$ as $[14]$;

$\psi_{i, j}(t)= \begin{cases}2^{d / 2} b_{j}\left(2^{d} t-i\right) & \frac{i}{2^{d}} \leq t<\frac{i+1}{2^{d}} \\ 0 & \text { otherwise }\end{cases}$

where $b_{j}(t)$ represents an orthonormal Bernstein polynomial of order $j$. On taking $j=7$ and dilation parameter $d=0$ with the help of these 8 orthonormal Bernstein polynomials, we can calculate 8 Bernstein polynomials orthonormal multiwavelets,

$\psi_{0,0}(t)=\left\{\begin{array}{ll}b_{0}(t), & 0 \leq t<1 \\ 0, & \text { otherwise }\end{array}, \psi_{0,1}(t)=\left\{\begin{array}{ll}b_{1}(t), & 0 \leq t<1 \\ 0, & \text { otherwise }\end{array}, \cdots \psi_{0,7}(t)=\left\{\begin{array}{ll}b_{7}(t), & 0 \leq t<1 \\ 0, & \text { otherwise }\end{array}\right.\right.\right.$,

which are shown in Figure 2. Similarly, we can get $\psi_{i, j}(t)$ for different order $j$ and dilation paramete $d$

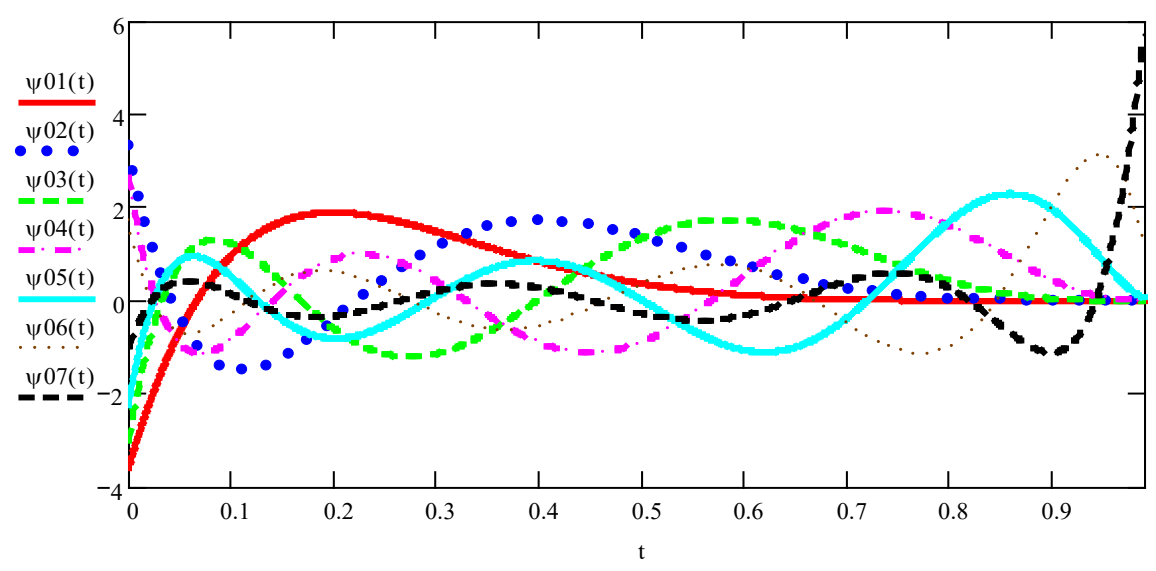

Figure 2 Eight Bernstein polynomials orthonormal multiwavelets for $j=7, d=0$.

\section{Function approximation}

As $f(t) \in L^{2}[0,1]$, we may expand $f(t)$ as:

$$
f(t)=\sum_{i=0}^{\infty} \sum_{j=0}^{\infty} e_{i j} \psi_{i j}(t)
$$

where $e_{i j}=\left\langle f(t), \psi_{i j}(t)\right\rangle$, here $\langle.,$.$\rangle denote the inner product on the Hilbert space L^{2}(R)$. In Eq. (6) the infinite series is truncated at the levels $i=2^{d}-1$ and $j=N$, we obtain an approximate representation for $f(t)$ as

$f(t) \approx \sum_{i=1}^{2^{d}-1} \sum_{j=0}^{N} e_{i j} \psi_{i j}(t)=E^{T} \Psi(t)$

where $E$ and $\Psi$ are $2^{d}(N+1) \times 1$ order matrices given by; 


$$
\begin{aligned}
& E=\left[e_{00}, \ldots, e_{0 N} ; e_{10}, \ldots, e_{1 N} ; \ldots ; e_{\left(2^{d}-1\right) 0}, \ldots, e_{\left(2^{d}-1\right) N}\right]^{T}, \\
& \Psi(t)=\left[\psi_{00}(t), \ldots, \psi_{0 N}(t) ; \psi_{10}(t), \ldots, \psi_{1 N}(t) ; \ldots ; \psi_{\left(2^{d}-1\right) 0}(t), \ldots, \psi_{\left(2^{d}-1\right) N}(t)\right]^{T} .
\end{aligned}
$$

Bernstein polynomials multiwavelets operational matrix of integration

In this section, the operational matrix of integration $P$ is derived. First, we find the matrix $P$ then on transforming the differential equation into integral equations by integration, after that truncating orthogonal series and approximating various signals involved in the equation by using the operational matrix of integration to remove the integral operations. The elements are the basis functions, orthogonal on a certain interval $[a, b]$. The Haar wavelets-based operational matrix of integration was derived by [1]. The integration of $\Psi(t)$ is approximated by Bernstein polynomials orthonormal multiwavelets series by Bernstein polynomials orthonormal multiwavelets coefficient matrix $P$;

$$
\int_{0}^{t} \Psi(t) d t=P_{2^{d}(N+1) \times 2^{d}(N+1)} \Psi(t),
$$

where $P$ is $2^{d}(N+1)$-order Bernstein polynomials orthonormal multiwavelets based operational matrix of integration.

\section{Method of solution}

In this section, we find the solution of the differential equation,

$L \frac{d^{2} q}{d t^{2}}+R \frac{d q}{d t}+\frac{q}{C}=V(t)$

governing the flow of current in a series LCR circuit is given subject to an applied voltage $V(t)=V_{0} \sin \omega t$ with boundary conditions $q(t)=0$ and $q^{\prime}(t)=0$. On integrating Eq. (1) with respect to $t$ and taking $V(t)=D^{T} \psi(t)$ we get;

$L \int_{0}^{t} \frac{d^{2} q}{d t^{2}} d t+R \int_{0}^{t} \frac{d q}{d t} d t+\int_{0}^{t} \frac{q}{C} d t=\int_{0}^{t} D^{T} \psi(t) d t$

on taking

$q(t)=E^{T} \psi(t)$

(10)

we get

$L \int_{0}^{t} \frac{d^{2} q}{d t^{2}} d t+R \int_{0}^{t} \frac{d q}{d t} d t+\frac{1}{C} \int_{0}^{t} q d t=\int_{0}^{t} D^{T} \psi(t) d t$,

(11)

on using Eqs. (8) and (10) with boundary conditions Eq. (11) becomes;

$L \frac{d q}{d t}+R E^{T} \psi(t)+\frac{1}{C} E^{T} P \psi(t)=D^{T} P \psi(t)$,

(12)

on integrating Eq. (12) with respect to $t$; 
$L \int_{0}^{t} \frac{d q}{d t} d t+R E^{T} \int_{0}^{t} \psi(t) d t+\frac{1}{C} E^{T} P \int_{0}^{t} \psi(t) d t=D^{T} P \int_{0}^{t} \psi(t) d t$,

again, using Eqs. (8) and (10) with boundary conditions;

$L E^{T} \psi(t)+R E^{T} P \psi(t)+\frac{1}{C} E^{T} P^{2} \psi(t)=D^{T} P^{2} \psi(t)$,

$L E^{T}+R E^{T} P+\frac{1}{C} E^{T} P^{2}=D^{T} P^{2}$,

$X E=Z$,

where $X=L I+R(P)^{T}+\frac{1}{C}\left(P^{2}\right)^{T} ; Z=\left(P^{2}\right)^{T} D$,

and $I$ stands for the identity matrix. Eq. (16) is a set of algebraic equations which can be solved for $E$, where $P$ is $2^{d}(N+1)$ order square matrix, called Bernstein multiwavelets-based operational matrix. On putting the values of $E$ into Eq. (10), we get the desired solution. Similarly, in the LC circuit case, we can obtain current by integrating Eq. (2) with respect to $t$ and using initial conditions (see Appendix).

\section{Illustrative examples}

The subsequent examples are illustrated to show the effectiveness and steadiness of our algorithm. Note that in the first example, the series in Eq. (7) is truncated at the level $j=5$ and $j=7$, respectively in example 1, 2 and example 3. The exactness of the proposed method is shown by manipulating the absolute error. $\Delta \xi\left(t_{k}\right)$, which is given by:

$\mathrm{E}(t)=\left|\xi\left(t_{k}\right)-\tilde{\xi}\left(t_{k}\right)\right|$,

where $\tilde{\xi}\left(t_{k}\right)$ is the approximate solution calculated at the point $t_{k}$ and the exact solution at the corresponding point is $\xi\left(t_{k}\right)$.

\section{Example 1}

Consider the differential equation governing the LCR circuit given in Eq. (1) with $L=1 \mathrm{H}, C=1 F, R=1 \Omega$ and the source voltage $V(t)=V_{0} \sin (\omega t)$ is:

$L \frac{d^{2} q}{d t^{2}}+R \frac{d q}{d t}+\frac{q}{C}=V_{0} \sin (\omega t)$

where $V_{0}=1 \mathrm{~V}, \omega=2$ with boundary conditions $q(t)=0$ and $q^{\prime}(t)=0$, whose exact solution is given by $q(t)=\frac{1}{13}\left[e^{-t / 2}\left(2 \cos \left(\frac{\sqrt{3}}{2} t\right)+\frac{14 \sqrt{3}}{3} \sin \left(\frac{\sqrt{3}}{2} t\right)\right)-2 \cos 2 t-3 \sin 2 t\right]$

Now, on using Bernstein polynomial multiwavelets operational matrix of integration we solve the above LCR circuit problem using Eqs. (9) to (16) and calculated the approximated solution $q 1(t)$ and $q 2(t)$ for $J=5 ; d=0$. Figures 3 and $\mathbf{4}$ are representing the exact and approximate solution and absolute error $\mathrm{E} 1(t)=|q(t)-q 1(t)|$. 


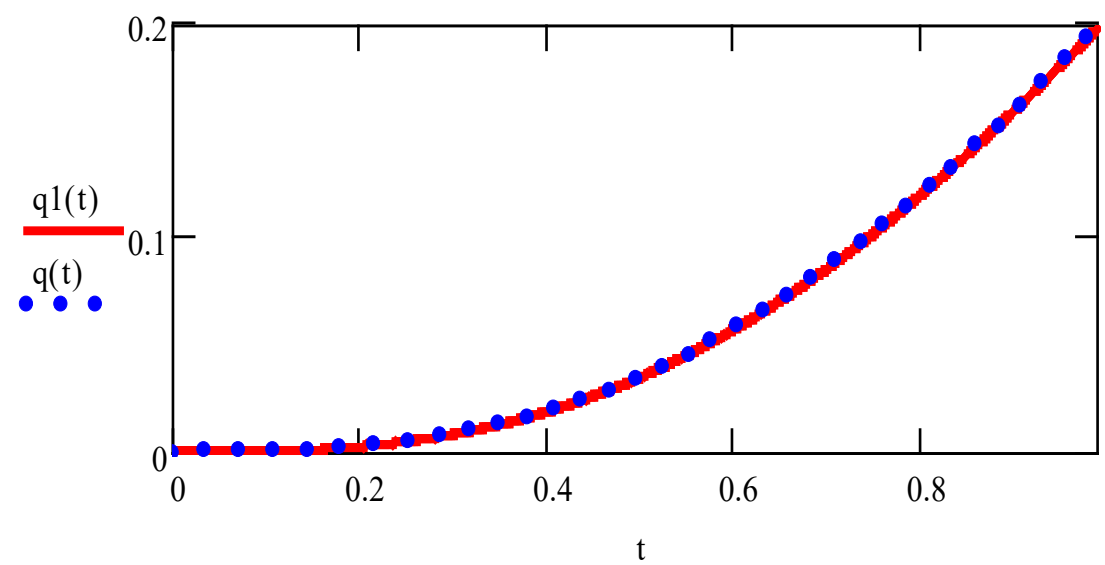

Figure 3 Comparison of solutions; exact solution $q(t)$; approximated solution $q 1(t)$.

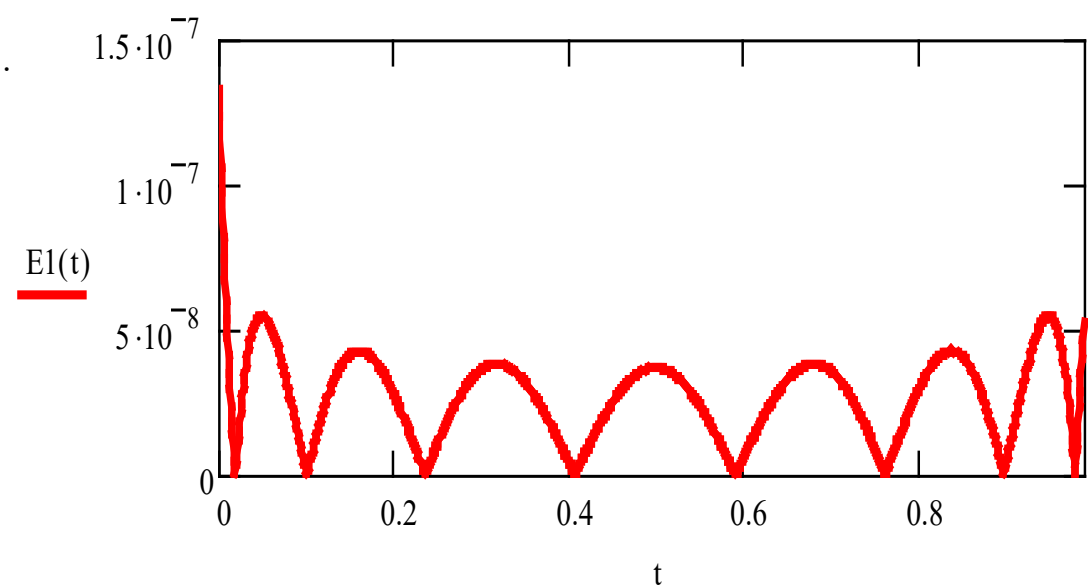

Figure 4 Absolute error $E 1(t)$ for $J=5 ; d=0$.

Table 1 Approximate and exact solution of example 1.

\begin{tabular}{cccl}
\hline $\boldsymbol{p}$ & $\begin{array}{c}\text { Exact solutions } \\
q(t)\end{array}$ & $\begin{array}{c}\text { Approximate } \\
\text { solution } \\
q 1(t)\end{array}$ & $\begin{array}{c}\text { Absolute } \\
\text { error } \\
E 1(t)=|q(t)-q 1(t)|\end{array}$ \\
\hline 0.0 & $3.243478 \times 10^{-4}$ & $3.243457 \times 10^{-4}$ & $2.092873 \times 10^{-9}$ \\
0.2 & $2.512962 \times 10^{-3}$ & $2.512991 \times 10^{-3}$ & $2.844036 \times 10^{-8}$ \\
0.4 & 0.018583 & 0.018583 & $5.415651 \times 10^{-9}$ \\
0.6 & 0.056811 & 0.056811 & $5.226375 \times 10^{-9}$ \\
0.8 & 0.119373 & 0.119373 & $2.857198 \times 10^{-8}$ \\
0.99 & 0.201866 & 0.201866 & $1.323026 \times 10^{-7}$ \\
\hline
\end{tabular}

\section{Example 2}

Consider the LC circuit given in Eq. (2) with $L=5 \mathrm{H}, C=.2 F, \omega=5$, the source voltage is $V(t)=V_{0} \cos (\omega t)$, where $V_{0}=1 \mathrm{~V}$ with boundary conditions $i(t)=0$ and $i^{\prime}(t)=0$ and the exact solution is given by $i(t)=(5 \sin (t)-\sin (5 t)) / 24$. 
Now using Bernsteins polynomial multiwavelets operational matrix of integration, we solve this LC circuit problem and calculate approximated solutions $i 1(t)$ and $i 2(t)$ for $d=0$ and $d=1$, respectively by utilizing the above algorithm from Eqs. (9) to (16). Figure 5 represents the exact and approximate solution. Figure 6 accounts for absolute errors; $\mathrm{E} 1(t)=|\mathrm{i}(t)-i 1(t)|$ and $\mathrm{E} 2(t)=|\mathrm{i}(t)-i 2(t)|$ for dilation parameter $d=0$ and $d=1$, respectively.

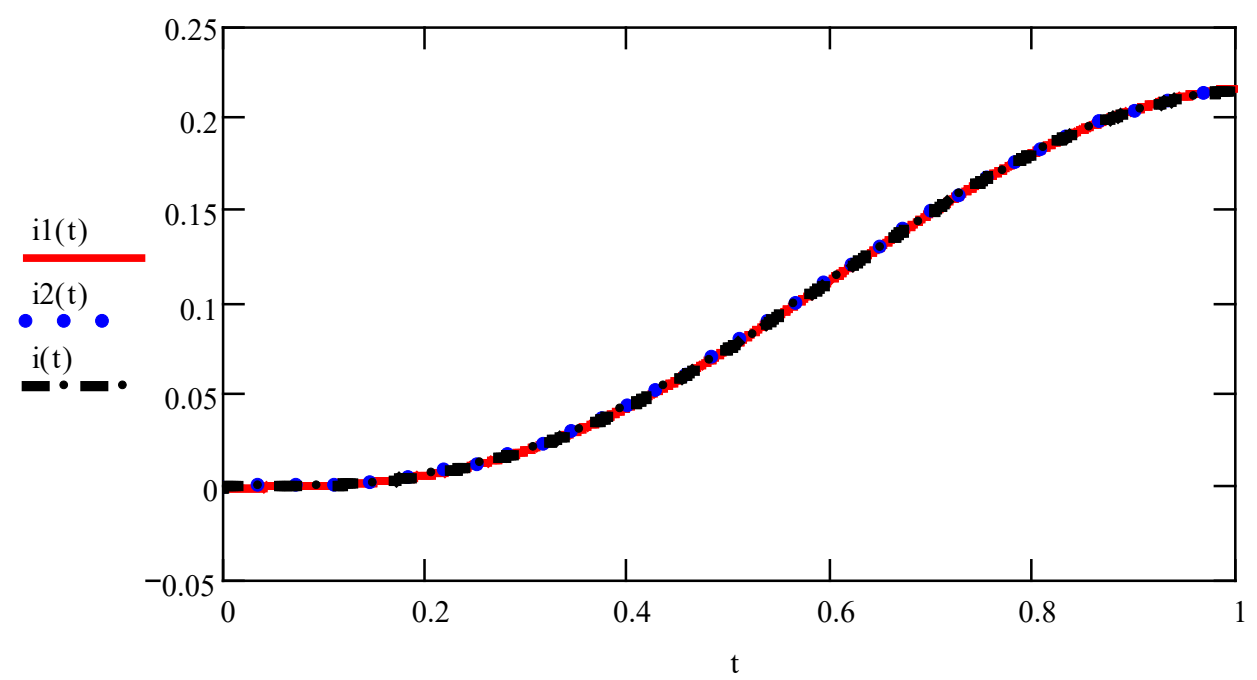

Figure 5 Comparison of solutions; exact solution $i(t)$; approximated solution $i 1(t)$ (with dilation parameter $d=0$ ) and $i 2(t)$ (with dilation parameter $d=1$ ).

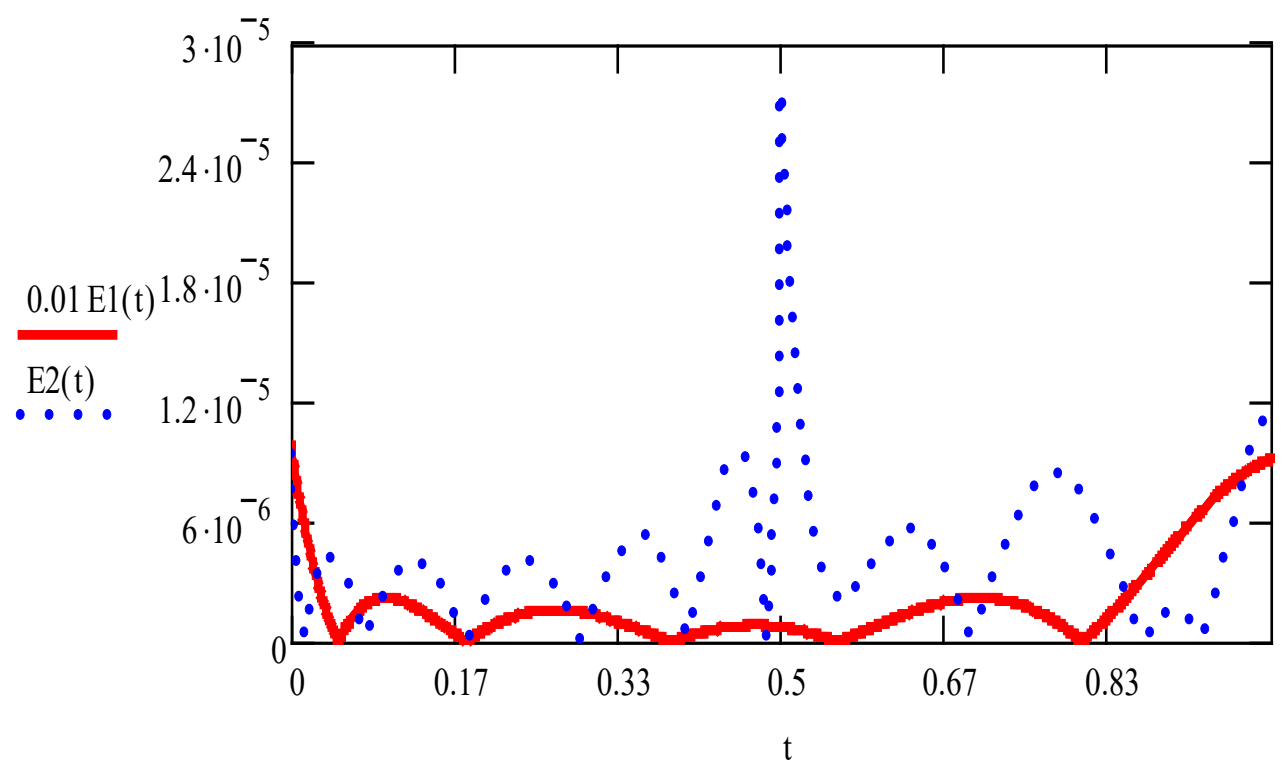

Figure 6 Comparison of absolute errors; $E 1(t)$ and $E 2(t)$ for dilation parameter $d=0$ and $d=1$ 
Table 2 Approximate and exact solution of example 2.

\begin{tabular}{cccc}
\hline $\boldsymbol{p}$ & $\begin{array}{c}\text { Exact solutions } \\
i(p)\end{array}$ & $\begin{array}{c}\text { Approximate } \\
\text { solution } \\
i 1(p) \text { (for } d=0)\end{array}$ & $\begin{array}{c}\text { Approximate } \\
\text { solution } \\
i 2(p) \text { (for } d=1)\end{array}$ \\
\hline 0.0 & 0.0 & 0.000000 & -0.000000 \\
0.2 & 0.006328 & 0.006263 & 0.006330 \\
0.4 & 0.043241 & 0.043260 & 0.043240 \\
0.6 & 0.111754 & 0.111669 & 0.111758 \\
0.8 & 0.180983 & 0.180955 & 0.180975 \\
0.99 & 0.215262 & 0.216178 & 0.215250 \\
\hline
\end{tabular}

\section{Example 3}

Consider the problem,

$\frac{1}{2} y^{\prime}(p)+y(p)=u(p)$

with $y(0)=0$

where $u(p)$ is $p^{4}$. The analytic solution of Eq. (18) is

$y(p)=\frac{3}{2}(1-\exp (-4 p))+p^{3}(p-2)+3 p(p-1)$.

We solve this by Bernstein polynomials multiwavelets, with $j=7 ; d=0$. We assume the unknown function $y(p)$ is given by $y(p)=C^{T} \psi(p)$, to get the solution of the differential equation; take

$y(p)=C^{T} \psi(p), u(p)=D^{T} \psi(p)$.

On integrating Eq. (18), we get

$\frac{1}{2} C^{T} \psi(p)=-\int_{0}^{p} C^{T} \psi(p) d p+\int_{0}^{p} D^{T} \psi(p) d p$,

$\frac{1}{2} C^{T}+C^{T} P=D^{T} P$

$L C=M$,

where $L=\frac{1}{2} I+P^{T} ; M=P^{T} D$ and $I$ is the identity matrix Eq. (19) is a set of algebraic equations which gives the matrix $C$ where $P$ is $2^{d}(N+1)$ order square matrix, called Bernstein multiwaveletsbased operational matrix. After getting the value of $C$, we get the desired solution. Figure 7 and 8 represents the approximate solution and absolute errors, respectively. 
Table 3 Approximate and exact solution of example 3.

\begin{tabular}{cccc}
\hline $\boldsymbol{p}$ & $\begin{array}{c}\text { Exact solution } \\
\xi 1(p)\end{array}$ & $\begin{array}{c}\text { Approximate } \\
\text { solutions } \\
\xi 2(p)\end{array}$ & $\begin{array}{c}\text { Approximate error } \\
\mathrm{E}(p)=|\xi 1(p)-\xi 2(p)|\end{array}$ \\
\hline 0.0 & 0.0 & $2.814345 \times 10^{-7}$ & $2.814345 \times 10^{-7}$ \\
0.2 & $1.199309 \times 10^{-4}$ & $1.199842 \times 10^{-4}$ & $5.328864 \times 10^{-8}$ \\
0.4 & $3.606554 \times 10^{-3}$ & $3.606552 \times 10^{-3}$ & $2.230206 \times 10^{-9}$ \\
0.6 & 0.025809 & 0.025809 & $1.983451 \times 10^{-8}$ \\
0.8 & 0.102755 & 0.102755 & $6.534773 \times 10^{-8}$ \\
0.99 & 0.283194 & 0.283194 & $1.156281 \times 10^{-7}$ \\
\hline
\end{tabular}

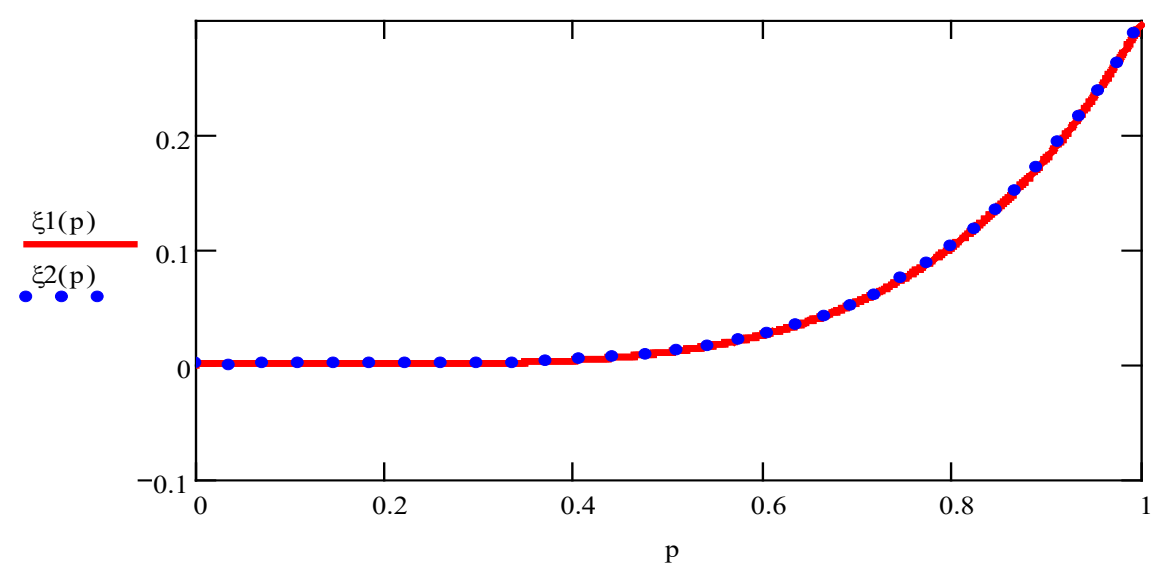

Figure 7 Comparison of solutions; exact solution $\xi 1(p)$; approximated solution $\xi 2(p)$ (for $d=0$ ).

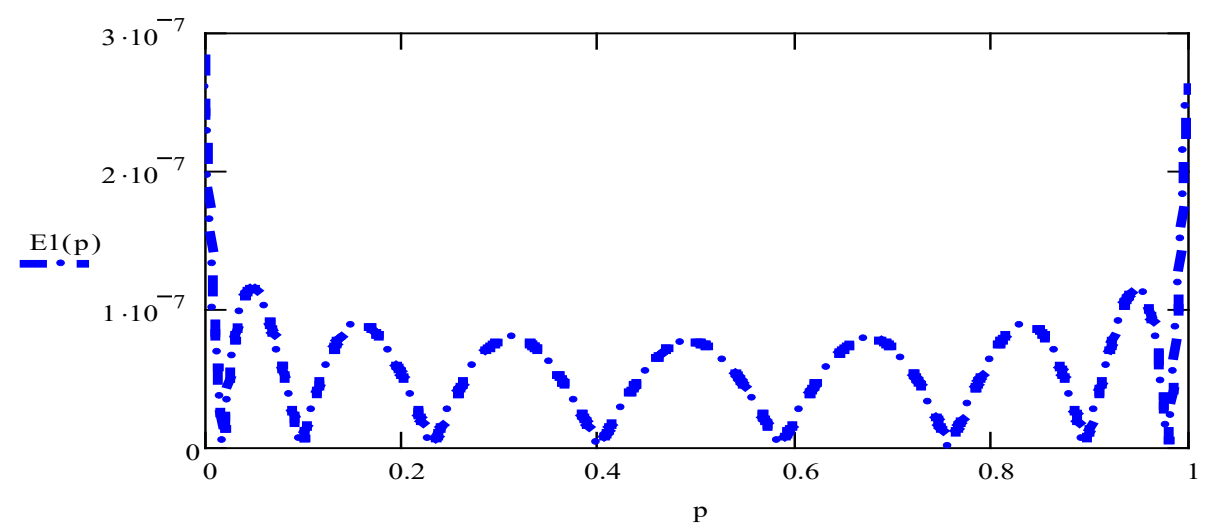

Figure 8 Absolute error $E(\mathrm{t})$ for $j=7, d=0$.

\section{Conclusions}

We have used Bernstein polynomials multiwavelets to construct the operational matrix of integration with 2 dilation parameters $d=0$ and $d=1$. Furthermore, our method illustrates the comparison of 2 distinct dilation parameters' solutions. The method is simple and clear due to the use of only 6 orthonormal polynomials of degree 5 . 


\section{References}

[1] JS Guf and WS Jiang. The Haar wavelets operational matrix of integration. Int. J. Syst. Sci. 1996; 27, 623-8.

[2] MHT Alshbool, AS Bataineh, I Hashim and OR Isik. Solution of fractional-order differential equations based on the operational matrices of new fractional Bernstein functions. J. King Saud Univ. Sci. 2017; 29, 1-18.

[3] Y Ozturk. Numerical solution of systems of differential equations using operational matrix method with Chebyshev polynomials. J. Taibah Univ. Sci. 2018; 12, 155-62.

[4] L Shi, X Ding, Z Chen and Q Ma. A new class of operational matrices method for solving fractional neutral pantograph differential equations. Adv. Differ. Equat. 2018; 2018, 94.

[5] ASVR Kanth and NU Kumar. A haar wavelet study on convective-radiative fin under continuous motion with temperature-dependent thermal conductivity. Walailak J. Sci. Tech. 2014; 11, 211-24.

[6] AZIZ Imran, SU Islam, M Fayyaz and M Azram. New algorithms for numerical assessment of nonlinear integro-differential equations of second-order using haar wavelets. Walailak J. Sci. Tech. 2015; 12, 995-1007.

[7] M Mehra. Wavelets and differential equations a short review. AIP Conf. Proc. 2009; 1146, 241-52.

[8] S Pandey, S Dixit and SR Verma. Bernstein polynomial multiwavelets operational matrix for solution of differential equation. In: N Deo, V Gupta, A Acu and P Agrawal (Eds.). Mathematical analysis I: Approximation theory. Springer, Singapore, 2018, p. 37-46.

[9] JO Stromberg. A modified Franklin system and higher-order spline systems on Rn as unconditional bases for Hardy spaces. In: Proceedings of the Harmonic Analysis, University of Chicago, 1981, p. 475-94.

[10] A Grossmann and J Morlet. Decomposition of hardy functions into square integrable wavelets of constant shape. SIAM J. Math. Anal. 1984; 15, 723-36.

[11] I Daubechies. Orthonormal bases of compactly supported wavelets. Comm. Pure Appl. Math. 1988, 41, 909-96.

[12] SG Mallat. A theory for multiresolution signal decomposition: The wavelet representation. IEEE Trans. Pattern Anal. Mach. Intell. 1989; 7, 674-93.

[13] Y Meyer. Analysis at Urbana 1: Analysis in function spaces. Cambridge University Press, Cambridge, 1989.

[14] SA Yousefi. B-polynomial multiwavelets approach for the solution of Abel's integral equation. Int. J. Comput. Math. 2010; 87, 310-6. 


\section{Appendix: Algorithm for the numerical method}

Step1: Obtain the orthonormalized version $b_{n}(u)$ of Bernstein polynomials $B_{j, n}(u)$.

1) The Bernstein polynomial of degree $N$ is $B_{j, N}(y)$

$$
b_{0}(y):=\frac{B_{0, N}(y)}{\left\|B_{0, N}(y)\right\|} .
$$

2) For $j=1$ to $N$

$$
\begin{aligned}
C_{j, N}(y):= & B_{j, N}(y)-\frac{\left\langle B_{j, N}(y), \mathrm{C}_{j-1, N}(y)\right\rangle}{\left\|\mathrm{C}_{j-1, N}(y)\right\|} * \mathrm{C}_{j-1, N}(y)-\frac{\left\langle B_{j, N}(y), \mathrm{C}_{j-2, N}(y)\right\rangle}{\left\|\mathrm{C}_{j-2, N}(y)\right\|} * \mathrm{C}_{j-2, N}(y) \\
& \ldots-\frac{\left\langle B_{j, N}(y), B_{0, N}(y)\right\rangle}{\left\|B_{0, N}(y)\right\|} * B_{0, N}(y) .
\end{aligned}
$$

3)

$$
b_{j}(y):=\frac{C_{j, N}(y)}{\left\|C_{j, N}(y)\right\|}
$$

is the required Bernstein polynomial. Here $\langle p(y), q(y)\rangle$ represents the inner product of $p(y)$ and $q(y)$ in the Hilbert space $L^{2}(R)$ and $\|\xi(y)\|$ is the $L^{2}$ norm of the given function $\xi(y)$.

Step2: Evaluate $\psi_{i, j}(t)$ over the interval $[0,1)$ using $b_{j}(t)$ obtained from step 1.

Step3: Manipulate operational matrices $P$ and matrix $D^{T}$.

Step4: On integrating given differential equation governing AC circuit with respect to $t$ and taking Voltage function $V(t)=D^{T} \psi(t), q(t)=E^{T} \psi(t)$ and $\int_{0}^{t} q d t=E^{T} P \psi(t)$ it converts to an algebraic system which can be easily solved for $E$.

Step5: Put values of $E^{T}$ from step 4 to get the desired solution function is $q(t)=E^{T} \psi(t)$. 